\title{
Vorwort für die erste Auflage
}

Es gibt wohl kein Handwerk, welches für die Herstellung seiner Erzeugnisse so viele Werkstoffe verarbeitet, wie es beim Orthopädiemechaniker- und im Schuhmacher-Handwerk aus der Vielseitigkeit ihrer Aufgaben geschieht. Dazu treten in der Gegenwart noch die von Wissenschaft und Technik neu entwickelten Werkstoffe, die wir als Kunststoffe kennen.

In meiner langjährigen Tätigkeit auf dem Gebiete der Orthopädietechnik habe ich oft erkennen müssen, daß hinsichtlich der Werkstoffkunde noch manche Wissenslücke offensteht, die ihre Ursache nicht zuletzt in der Tatsache hat, daß dem Fachhandwerker keine ausreichende Literatur über seine Werkstoffe zur Verfügung steht. So war es Zweck und Ziel dieser Arbeit, die in diesem Handwerk zur Verarbeitung gelangenden Werkstoffe hinsichtlich ihres Ursprungs, ihrer Gewinnung bzw. Herstellung, ihrer Eigenschaften und ihrer Verwendungsmöglichkeiten zusammenzustellen.

Ich habe mich bemüht, den Fragenkomplex des Themas in einfacher und knapper Sprache zu behandeln, damit dem Leser die Möglichkeit gegeben ist, sich einen kurzen und verständlichen Überblick zu verschaffen.

Berlin, Sommer 1954

Fritz Püschel

\section{Vorwort für die zweite Auflage}

Die vorliegende zweite Auflage des Buches über Werkstoffe im Orthopädieund Schuhmacher-Handwerk hat eine Erweiterung dahingehend erfahren, daß nunmehr auch die Werkstoffe mehr als bisher berücksichtigt worden sind, die im Schuhmacherhandwerk verarbeitet werden.

Weiter wurde den verschiedenen neuzeitlichen Kunststoffen größerer Platz gegeben, weil sie sich gerade in den letzten Jahren erfolgreich eingeführt und bewährt haben.

Darüber hinaus wurde bei den verschiedenen natürlichen und den synthetisch hergestellten Werkstoffen (Kunststoffen) auf die Anwendungsmöglichkeiten hingewiesen und auch die jeweils geltenden Verarbeitungs- und Verformungsmethoden eingehend beschrieben.

Ich hoffe, damit dem Leser des Buches einen ausführlichen Ưberblick über das gestellte Thema zu geben.

Berlin, Sommer 1961

Fritz Püschel 